\title{
Structural and Dynamic Analysis of a Thioredoxin-motif containing protein from the Conjugative F plasmid of Escherichia coli
}

\author{
Gerald F. Audette \\ Dept. of Chemistry \& The Centre for Research on Biomolecular Interactions \\ York University, 4700 Keele St., Toronto, ON, M3J 1P3
}

The transfer of genetic material within a bacterial population through the process of conjugation is an evolutionarily conserved tactic by which novel genetic elements are distributed for survival in unique environments. The Escherichia coli F-plasmid houses all genes necessary for the assembly of its conjugative machinery to facilitate successful DNA transfer. These genes encode proteins that facilitate or make up parts of the F-conjugation complex including; pilus formation, mating pair formation (Mpf), DNA transfer complex and exclusion factors. The transfer proteins TraF and TrbB are hallmark proteins of F-like, and both contain thioredoxin motifs. Indeed recent bioinformatics and biochemical evidence indicates that $\operatorname{TrbB}$ is a disulfide bond isomerase closely related to DsbC. However TraF, essential for the assembly of the conjugative F pilus, does not contain the active site cysteines of thioredoxins; it retains an as yet undefined function independent of redox activity. While it is apparent that TrbB plays a role in the redox chemistry of the F T4SS, how TraF functions remains unclear. In order to characterize the structural differences between these two thioredoxin-motif containing T4SS proteins, TraF has been analyzed crystallographically (space group $\mathrm{C} 2$; $\mathrm{a}=119.87 \AA, \mathrm{b}=34.36 \AA, \mathrm{c}=46.21 \AA$ and $\beta=$ $90.40^{\circ}$, and crystals diffracted to $2.3 \AA$ resolution, and through time-resolved hydrogen deuterium exchange mass spectrometry. 